\title{
Asociación entre el índice de masa corporal y la talla desde el nacimiento hasta los 5 años en preescolares chilenos
}

\author{
JULIANA KAIN ${ }^{1, \mathrm{a}}$, CAMILA CORVALÁN ${ }^{2}$, LYDIA LERA ${ }^{1, \mathrm{~b}}$, \\ MARCOS GALVÁN ${ }^{3, c}$, GERARDO WEISSTAUB ${ }^{1}$, RICARDO UAUY ${ }^{1,4}$
}

${ }^{1}$ Instituto de Nutrición y

Tecnología de los Alimentos (INTA), Universidad de Chile. Santiago, Chile.

2Escuela de Salud Pública, Facultad de Medicina, Universidad de Chile. Santiago, Chile. 3Universidad Autónoma de Hidalgo, Pachuca, México. ${ }^{4}$ London School of Hygiene and Tropical Medicine, Londres, Inglaterra. aBioquímico, Master en Salud Pública. bEstadístico, PhD.

'Nutricionista, PhD

Recibido el 30 de junio de 2010, aceptado el 28 de marzo de 2011.

Correspondencia a: Juliana Kain

Instituto de Nutrición y Tecnología de los Alimentos (INTA)

Universidad de Chile. Santiago, Chile.

E-mail: jkain@inta.uchile.cl

\section{Association between body mass index (BMI) and height from birth to 5 years in Chilean preschool children}

Background: It has been shown that excess weight gain during childhood is associated with a greater risk of obesity; this relationship may be mediated by growth in height. Aim: To quantify the associations between $z$ scores for body mass index (BMIZ) and height for age (HAZ) from birth to 5 years of age and to assess how this association varies according to age and adiposity. Material and Methods: Weight and height were recorded at 11 occasions from birth on a cohort of 1089 Chilean five year-old children with a birth weight $>2500 \mathrm{~g}$. BMI, BMIZ, HAZ and prevalence of obesity were calculated. We determined the cross-sectional association between BMIZ and HAZ for the total sample and by 3 BMI Z categories, using Pearson correlation. We determined the probability of obesity according to four HAZ categories. Results: Obesity increased continuously reaching approximately $16 \%$ at 5 years. Stunting was virtually nonexistent. No association between BMIZ and HAZ was observed at birth, while at 1 and 2 months, leaner infants had lower stature. No association between these parameters was observed between 6-24 months, while after 3 years, a clear relationship was observed. Conclusions: The probability of a preschool child of being obese is less than $10 \%$ with a HAZ is $<0$. Nevertheless, children with heights above the median (even within the normal range) have an increased risk of obesity, especially after $3 y$ of age. Between 0-5 years, the relationship between weight and height varies by age and adiposity.

(Rev Med Chile 2011; 139: 606-612).

Key words: Body height; Body mass index; Child, preschool.
L a obesidad infantil está aumentando en forma alarmante tanto en países desarrollados como en desarrollo ${ }^{1}$. En Chile, la prevalencia de obesidad en niños de 6 años casi se ha triplicado durante las dos últimas décadas, desde 8,7 en 1989 a $21,8 \%{ }^{2}$ en el 2009. En la etapa escolar, los niños obesos además de tener más peso, son más altos que sus pares de peso normal. Freedman y $\mathrm{cols}^{3-5}$ determinaron la asociación entre el índice de masa corporal (IMC) y la estatura en niños norteamericanos entre 5 y 8 años, demostrando que el aumento en $1 \mathrm{DE}$ de la talla/edad está asociado con un aumento entre 2 y 4,6 veces en la prevalencia de obesidad en niños y niñas respectivamente.

La evidencia sobre una asociación entre talla e IMC en preescolares es escasa. Buchan y cols ${ }^{6}$ usando encuestas anuales transversales para examinar la evolución de la relación entre IMC y talla en niños ingleses de 3 años, desde 1988 al 2003, mostraron que esta asociación fue más fuerte con el tiempo. La correlación peso-talla aumentó desde 0,59 a 0,71 debido a que el IMC aumentó más en 
los niños más altos. Los autores concluyeron que "el aumento de adiposidad en niños pequeños implica tanto crecimiento como mayor apetito, es decir, que niños creciendo rápido y con más apetito están ahora más expuestos a un ambiente "obesogénico".

Stanojevic y cols ${ }^{7}$ determinaron la relación entre IMC y talla en preescolares entre 2 y 5 años asistentes a la Junta Nacional de Jardines Infantiles (JUNJI) desde 1996 a 2004 encontrando que la proporción de niños altos había aumentado progresivamente y que éstos presentaban menor prevalencia de obesidad. Kain y $\operatorname{cols}^{8}$, utilizando datos de peso y talla de aproximadamente $70 \%$ del universo de primer grado del país entre 1987 y 2002 (encuestas a cargo de la Junta Nacional de Auxilio Escolar y Becas), encontraron que existía una asociación entre talla y obesidad, cuya fuerza había aumentado en el tiempo.

Lo anterior indicaría que el IMC y la talla estarían asociados en forma diferente según la edad, grado de adiposidad y retardo en talla. Por lo tanto, el objetivo de este estudio fue cuantificar la asociación entre IMC y talla desde el nacimiento hasta los 5 años.

\section{Metodología}

\section{A. Sujetos}

En 2006 seleccionamos, de 54 jardines infantiles pertenecientes a JUNJI de la zona sur de Santiago de Chile, preescolares entre 2,6 y 4 años, con nacimiento único y peso de nacimiento $>2.500 \mathrm{~g}$. La muestra incluyó 1.195 niños; de los cuales, 1.089 (91\%) tenían mediciones de peso y talla desde el nacimiento hasta los 5 años. Los niños pertenecen a familias de ingresos medio-bajos y bajos.

El protocolo del estudio fue aprobado por la Dirección de JUNJI y el Comité de Ética del INTA. Se obtuvo consentimiento informado de los padres.

Mediciones antropométricas: Las mediciones de peso y talla desde 0-3 años se obtuvieron de los carnés de salud. Estos datos se registran en forma rutinaria en los controles de atención primaria. La calidad de estas mediciones fue certificada de acuerdo a varios criterios que sugiere la Organización Mundial de la Salud (OMS) como son su variabilidad, preferencia de dígitos y número de observaciones extremas, teniendo que ser eliminadas menos de $1 \%$ de las mediciones por implausibilidad. Adicionalmente, en una submuestra de 55 niños, evaluamos la concordancia de las mediciones registradas en los carnés de salud con las realizadas por nutricionistas del estudio, siendo todas superiores a 0,82 .

A los 52 y 60 meses, 4 nutricionistas entrenadas recogieron estos datos usando procedimientos estandarizados. El peso fue medido con una pesa portátil (SECA 770 ), precisión de $0,1 \mathrm{~kg}$, la talla con un estadiómetro portátil (Harpenden 603), precisión de $0,1 \mathrm{~cm}$. Se confirmó la confiabilidad intra e inter observador de estas mediciones (correlación intra-clase $\geq 0,75$ ). La talla de la madre fue medida (como parte de una encuesta que incluyó información socioeconómica, el embarazo, conducta alimentaria y hábitos de vida del hijo) por 4 entrevistadores cuando los niños tenían 52 meses, utilizando los mismos instrumentos que en los niños. La confiabilidad de estas mediciones ha sido comprobada recientemente en estas mismas mujeres (correlaciones intra-clase $=0,96$ ).

\section{B. Análisis de datos}

En base al calendario de controles médicos establecidos, consideramos el peso y la talla a las siguientes edades: $0,1,2,4,6,12,24,36$ meses y además a los 52 y 60 meses. (11 momentos). El $75 \%$ de los niños contaba con $\geq 7$ mediciones, el resto entre 3 y 6 . En cada edad se calculó el IMC $\left(\mathrm{kg} / \mathrm{m}^{2}\right)$, los puntajes $\mathrm{Z}$ de IMC y de talla/edad (Z IMC y Z TE) y la prevalencia de obesidad ( $Z$ IMC > 2), usando como referencias OMS $2006^{11}$ y OMS $2007^{12}$.

Utilizamos correlación parcial de Pearson para determinar la asociación transversal entre $Z$ IMC y Z TE desde 0-5 años, ajustando por edad y sexo. Se realizaron los análisis considerando la muestra total y grado de adiposidad ( 3 categorías de Z IMC, $<0,0-1$ y $>1$ ). No se consideraron más categorías, ya que a ciertas edades los $\mathrm{N}$ eran muy pequeños. Coeficientes de correlación menores a 0,3 se consideran muy pequeños, aquellos entre 0,3 y 0,5 moderados $^{13}$. Para determinar la relevancia de estas asociaciones en términos del exceso de adiposidad, calculamos la probabilidad de ser obeso en cada una de las edades cronológicas entre 0 y 5 años, según 4 categorías de Z TE (<-1, -1- 0, 0-1 y $>1$ ). Se usaron estos puntos de corte, porque el $\mathrm{N}$ de niños con $Z \mathrm{TE}<-2 \mathrm{y}>+2$, fue menos de $5 \%$ 
en todas las edades. La diferencia de distribución total de las prevalencias entre cada grupo se evaluó usando test de $\chi^{2}$, mientras que las diferencias específicas entre grupos se evaluaron usando el compprop macro de $\mathrm{SAS}^{14}$.

A partir de los resultados de las correlaciones, identificamos 4 períodos de vida temprana en que las asociaciones entre IMC y talla diferían: nacimiento, 1 y 2 meses, 6-24 meses, $\geq$ a 36 meses. En cada uno de estos períodos evaluamos la relación entre IMC y talla utilizando modelos de regresión lineal que permiten dar cuenta de la dependencia de medidas repetidas (Generalized LinearMixed Models) controlando el efecto del sexo, edad, y talla de la madre. Los análisis se realizaron usando el paquete estadístico Stata $10.1^{14}$ para la muestra total y estratificada por estado nutricional. No fue necesario realizar los análisis según sexo, ya que las interacciones por sexo no fueron estadísticamente significativas $(p>0,05)$.

\section{Resultados}

Las mediciones antropométricas de 0-5 años según sexo se presentan en la Tabla 1 . El promedio de peso de nacimiento fue $3,44(0,43) \mathrm{kg}$. En ambos sexos, el Z IMC aumentó progresivamente a partir del primer mes, especialmente entre $6 \mathrm{y}$ 12 meses y entre 3 y 4 años. En los hombres (con excepción de una leve disminución a los 2 años), la prevalencia de obesidad aumentó progresivamente desde el nacimiento; a los 5 años ésta fue de 18,8\%; en las niñas, la prevalencia disminuyó el primer mes, aumentando después hasta alcanzar $12,6 \%$ a los 5 años. En ambos sexos, el promedio de Z TE fue normal al nacer, sin embargo, se observó una caída significativa al mes $(-0,53$ y - 0,25 en hombres y mujeres respectivamente). Desde los 2 meses, aún cuando el Z TE aumentó, éste se mantuvo bajo el valor normal de la referencia hasta los 5 años siendo más notorio en los varones. En general la talla baja (ZTE <-2 o "stunting") no constituyó un problema en las niñas, sin embargo, en los varones, a ciertas edades ( 1,2 y 4 meses) esta proporción fue mayor a $5 \%$.

En la Tabla 2 se observan las correlaciones entre el Z IMC y Z TE en la muestra total y según 3 categorías de $\mathrm{Z}$ IMC. Al nacer, estas dos variables no se relacionaron, mientras que al mes, hubo una pequeña asociación directa, pero sólo en los niños más delgados, es decir, estos últimos presentaban una menor talla. No se observó asociación desde

Tabla 1. Evolución de algunas características antropométricas de la muestra de 0 a 5 años, por sexo

\begin{tabular}{|c|c|c|c|c|c|c|c|c|c|c|c|}
\hline Hombres & $0 \mathrm{~m}$ & $1 \mathrm{~m}$ & $2 \mathrm{~m}$ & $4 \mathrm{~m}$ & $6 \mathrm{~m}$ & $12 \mathrm{~m}$ & $18 \mathrm{~m}$ & $24 \mathrm{~m}$ & $36 \mathrm{~m}$ & $52 \mathrm{~m}$ & $60 \mathrm{~m}$ \\
\hline$N$ & 538 & 384 & 386 & 365 & 347 & 441 & 391 & 426 & 302 & 538 & 539 \\
\hline Z IMC & $\begin{array}{c}0,14 \\
(0,9)\end{array}$ & $\begin{array}{r}0,11 \\
(1,0)\end{array}$ & $\begin{array}{c}0,30 \\
(1,0)\end{array}$ & $\begin{array}{c}0,46 \\
(1,0)\end{array}$ & $\begin{array}{c}0,50 \\
(1,0)\end{array}$ & $\begin{array}{c}0,80 \\
(1,0)\end{array}$ & $\begin{array}{c}0,83 \\
(0,9)\end{array}$ & $\begin{array}{c}0,80 \\
(1,0)\end{array}$ & $\begin{array}{c}0,80 \\
(1,1)\end{array}$ & $\begin{array}{c}0,97 \\
(1,1)\end{array}$ & $\begin{array}{c}0,99 \\
(1,2)\end{array}$ \\
\hline$\% O B$ & 3,9 & 3,8 & 5,5 & 7,1 & 7,2 & 11,9 & 12,5 & 10,3 & 16,5 & 16,3 & 18,6 \\
\hline Z TE & $\begin{array}{c}0,12 \\
(0,9)\end{array}$ & $\begin{array}{l}-0,53 \\
(1,0)\end{array}$ & $\begin{array}{l}-0,61 \\
(1,0)\end{array}$ & $\begin{array}{l}-0,32 \\
(1,0)\end{array}$ & $\begin{array}{l}-0,35 \\
(1,0)\end{array}$ & $\begin{array}{l}-0,36 \\
(1,1)\end{array}$ & $\begin{array}{l}-0,38 \\
(1,1)\end{array}$ & $\begin{array}{l}-0,19 \\
(1,1)\end{array}$ & $\begin{array}{l}-0,26 \\
(0,96)\end{array}$ & $\begin{array}{l}-0,18 \\
(0,92)\end{array}$ & $\begin{array}{l}-0,03 \\
(0,94)\end{array}$ \\
\hline $\begin{array}{l}\% \text { Z TE } \\
<-2\end{array}$ & 1,9 & 7 & 7,8 & 5,2 & 2,8 & 5,2 & 4,0 & 2,3 & 2,5 & 1,8 & 1,5 \\
\hline Mujeres & Birth & $1 \mathrm{~m}$ & $2 \mathrm{~m}$ & $4 \mathrm{~m}$ & $6 \mathrm{~m}$ & $12 \mathrm{~m}$ & $18 \mathrm{~m}$ & $24 \mathrm{~m}$ & $36 \mathrm{~m}$ & $52 \mathrm{~m}$ & $60 \mathrm{~m}$ \\
\hline$N$ & 545 & 397 & 381 & 367 & 366 & 452 & 420 & 429 & 350 & 549 & 550 \\
\hline Z IMC & $\begin{array}{c}0,20 \\
(1,0)\end{array}$ & $\begin{array}{c}0,20 \\
(1,0)\end{array}$ & $\begin{array}{c}0,40 \\
(0,9)\end{array}$ & $\begin{array}{c}0,40 \\
(1,0)\end{array}$ & $\begin{array}{c}0,47 \\
(1,0)\end{array}$ & $\begin{array}{c}0,77 \\
(0,9)\end{array}$ & $\begin{array}{c}0,87 \\
(0,9)\end{array}$ & $\begin{array}{c}0,80 \\
(1,0)\end{array}$ & $\begin{array}{c}0,85 \\
(1,0)\end{array}$ & $\begin{array}{c}0,90 \\
(1,0)\end{array}$ & $\begin{array}{c}0,85 \\
(1,0)\end{array}$ \\
\hline$\% O B$ & 3,6 & 1,7 & 3,0 & 5,5 & 6,8 & 8,1 & 8,3 & 10,3 & 10,4 & 12,5 & 12,6 \\
\hline Z TE & $\begin{array}{c}0,28 \\
(0,9)\end{array}$ & $\begin{array}{l}-0,25 \\
(0,89)\end{array}$ & $\begin{array}{l}-0,3 \\
(0,91)\end{array}$ & $\begin{array}{l}-0,15 \\
(0,9)\end{array}$ & $\begin{array}{l}-0,06 \\
(0,86)\end{array}$ & $\begin{array}{l}-0,15 \\
(0,94)\end{array}$ & $\begin{array}{l}-0,16 \\
(0,95)\end{array}$ & $\begin{array}{l}-0,04 \\
(0,98)\end{array}$ & $\begin{array}{l}-0,21 \\
(0,92)\end{array}$ & $\begin{array}{l}-0,21 \\
(0,87)\end{array}$ & $\begin{array}{l}-0,06 \\
(0,86)\end{array}$ \\
\hline $\begin{array}{l}\% \text { Z TE } \\
<-2\end{array}$ & 0,9 & 1,5 & 2,3 & 2,6 & 0,8 & 1,5 & 3,0 & 2,0 & 1,9 & 2,3 & 0,9 \\
\hline
\end{tabular}


el mes hasta los 3 años, mientras que a los 4 y 5 años, la relación fue directa y de pequeña magnitud, aunque creciente en los niños con sobrepeso.

En la Tabla 3 se presentan los resultados de los análisis de regresión lineal multivariado para cada uno de los períodos identificados previamente con asociaciones diferenciales entre IMC y talla en los análisis de correlaciones, para la muestra total y según 3 categorías de Z IMC. Al nacer, la magnitud y dirección de las asociaciones depende del estado nutricional, siendo positiva en niños delgados e inversa en niños con sobrepeso u obesidad, entre uno y dos meses existe una asociación positiva sólo en niños delgados; entre 6 y 24 meses de edad, la talla es independiente del IMC, mientras que sobre los 3 años de edad existe una asociación positiva, principalmente en los niños con sobrepeso $\mathrm{u}$ obesidad.
La Figura 1 muestra la prevalencia de obesidad entre 0 y 5 años, según categorías de Z TE. Se observa (con excepción de los 36 meses), que los niños con $\mathrm{Z} \mathrm{TE}<0$ tuvieron una probabilidad de $10 \%$ o menos de ser obesos; en aquellos con $\mathrm{Z} \mathrm{TE}$ entre 0 y 1 , esta probabilidad fue menor a $15 \%$ hasta los 36 meses, sin embargo, aumentó a $22 \%$ a los 5 años. En niños con Z TE > 1, la probabilidad de obesidad aumentó $2 \%$ desde el nacimiento a 31\% a los 5 años. La distribución de las prevalencias de obesidad fueron estadísticamente significativas en los dos grupos con $\mathrm{Z} \mathrm{TE}>0$. El análisis post-hoc mostró que en el grupo con $Z \mathrm{TE}$ entre 0 y 1 , las diferencias se debían principalmente a la baja prevalencia de obesidad al nacer, mientras que aquellos con $\mathrm{Z}$ TE $>1$ se encontraron diferencias significativas entre la prevalencia de obesidad a los 4 y 5 años y aquella al nacer, a los 12 y 24 meses.

Tabla 2. Correlación entre los puntajes $Z$ de talla y de IMC desde 0 a 5 años según categorías de $Z$ IMC

\begin{tabular}{|cccccccccc|}
\hline $\begin{array}{c}\text { Edad } \\
\text { (meses) }\end{array}$ & $\mathbf{Z}$ & $\mathbf{Z}$ IMC $<\mathbf{0}$ & \multicolumn{2}{c}{$\mathbf{0}<\mathbf{Z}$ IMC $\leq \mathbf{1}$} & \multicolumn{2}{c|}{$\mathbf{Z}$ IMC $>\mathbf{1}$} & \multicolumn{2}{c|}{ Todos } \\
\hline 0 & 501 & $\mathbf{r}$ & $\mathbf{n}$ & $\mathbf{r}$ & $\mathbf{n}$ & $\mathbf{r}$ & $\mathbf{n}$ & $\mathbf{r}$ \\
\hline 1 & 327 & $0,26^{*}$ & 321 & 0,08 & 155 & 0,04 & 803 & $0,23^{*}$ \\
\hline 2 & 266 & 0,04 & 329 & 0,08 & 199 & $-0,04$ & 794 & 0,09 \\
\hline 6 & 243 & $-0,13$ & 274 & $-0,05$ & 227 & 0,05 & 744 & 0 \\
\hline 12 & 195 & 0,07 & 370 & $-0,03$ & 366 & 0,03 & 931 & 0,04 \\
\hline 24 & 177 & 0,03 & 350 & $-0,06$ & 361 & 0,14 & 889 & 0,06 \\
\hline 36 & 146 & 0,1 & 246 & 0,01 & 284 & 0,12 & 676 & 0,07 \\
\hline 52 & 197 & 0,03 & 451 & 0,02 & 494 & $0,25^{*}$ & 1.142 & $0,23^{*}$ \\
\hline 60 & 223 & 0,03 & 419 & 0,08 & 449 & $0,30 *$ & 1.091 & $0,26^{*}$ \\
\hline
\end{tabular}

*Correlación de Pearson.

Tabla 3. Medidas repetidas Z IMC sobre Z TE en 4 períodos de crecimiento temprano

\begin{tabular}{|c|c|c|c|c|c|c|c|c|c|c|c|c|c|c|c|c|}
\hline \multirow{2}{*}{$\begin{array}{l}\text { Edad } \\
\text { (mes) }\end{array}$} & \multicolumn{4}{|c|}{$\mathrm{Z}$ IMC $<0$} & \multicolumn{4}{|c|}{$0<Z$ IMC $\leq 1$} & \multicolumn{4}{|c|}{ Z IMC > 1} & \multicolumn{4}{|c|}{ Todos } \\
\hline & n & B & SE & $\mathbf{P}$ & $\mathbf{n}$ & B & SE & $\mathbf{P}$ & $\mathbf{n}$ & B & SE & $\mathbf{P}$ & $\mathbf{n}$ & B & SE & $\mathbf{P}$ \\
\hline 0 & 461 & 0,16 & 0,07 & 0,02 & 396 & 0,22 & 0,16 & 0,2 & 195 & $-0,29$ & 0,12 & 0,02 & 1.052 & 0,07 & 0,03 & 0,02 \\
\hline $1-2$ & 321 & 0,37 & 0,10 & 0,0002 & 371 & 0,09 & 0,16 & 0,6 & 197 & 0,14 & 0,12 & 0,2 & 889 & 0,17 & 0,03 & $<0,0001$ \\
\hline $6-24$ & 337 & $-0,07$ & 0,07 & 0,3 & 431 & $-0,03$ & 0,08 & 0,7 & 301 & 0,13 & 0,04 & 0,0009 & 1.069 & 0,04 & 0,01 & 0,05 \\
\hline$\leq 36$ & 203 & 0,04 & 0,09 & 0,7 & 388 & 0,15 & 0,09 & 0,1 & 477 & 0,27 & 0,03 & $<0,0001$ & 1.068 & 0,19 & 0,02 & $<0,0001$ \\
\hline
\end{tabular}

*Modelos ajustados edad, sexo, talla materna. 


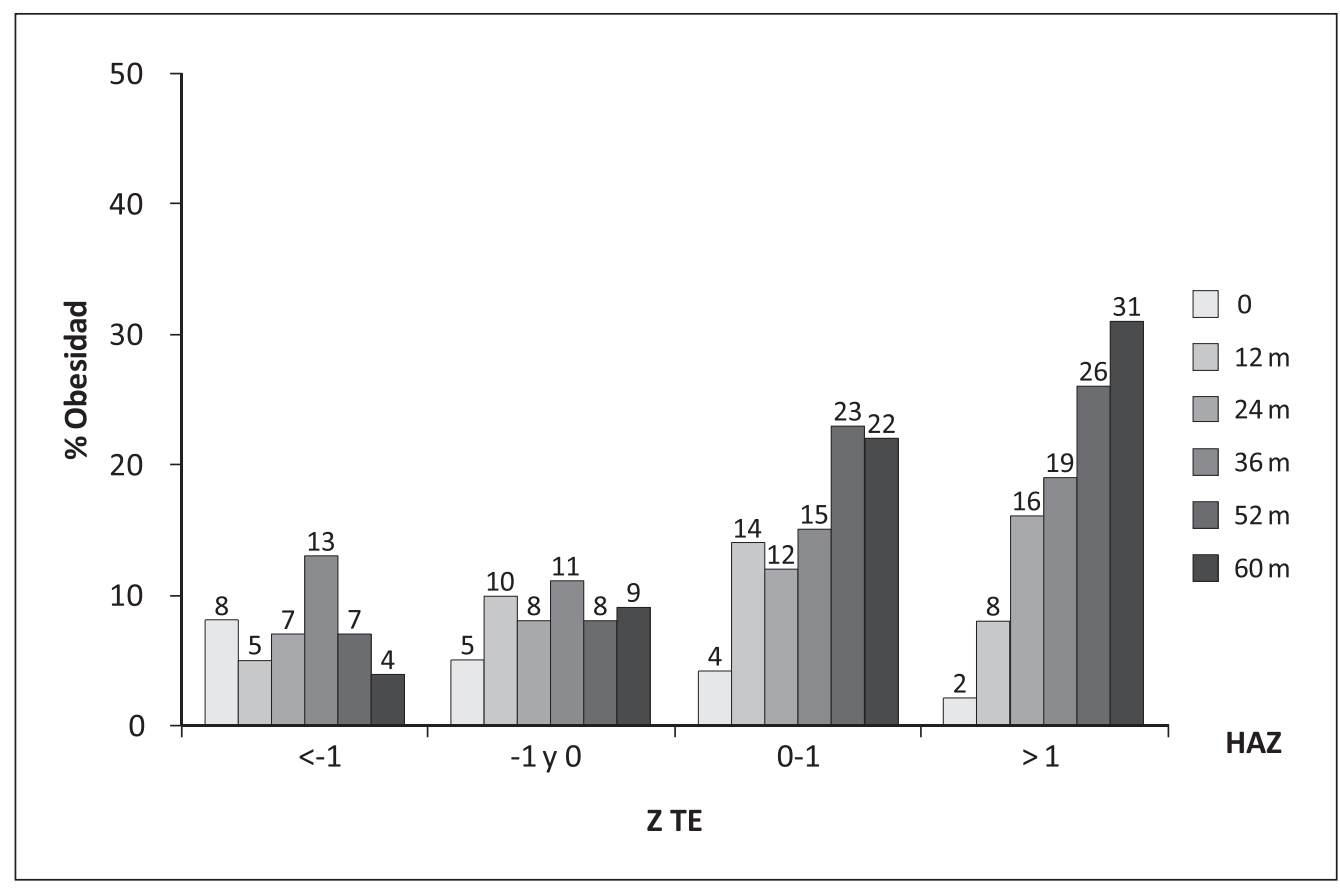

Figura 1.

\section{Discusión}

En este estudio se mostró que las asociaciones entre IMC y talla varían con la edad y el grado de adiposidad. Al nacer, no se observó asociación entre las dos variables; en los primeros dos meses, aquellos niños más delgados tenían una menor estatura; hasta los 3 años no se observó asociación, mientras que después, se observó una clara relación entre mayor adiposidad y estatura. Adicionalmente se mostró que preescolares con estatura bajo la mediana de la referencia OMS, tenían una baja probabilidad de ser obesos, sin embargo, aquellos niños con estatura sobre la mediana (aun en el rango normal), tenían una probabilidad alta de desarrollar obesidad. Este efecto fue más notorio en los niños con $\mathrm{Z} \mathrm{TE}>1$ a los 4 y 5 años.

Estos hallazgos muestran que las intervenciones para prevenir la obesidad infantil deberían comenzar tempranamente, idealmente desde los 2 años. Estrategias dirigidas a disminuir el IMC en niños obesos, podrían frenar la velocidad de crecimiento en talla, lo que no significa que tendrían un retardo en talla y tampoco una menor talla en la etapa adulta, tal como lo demostraron He y Karlberg ${ }^{15}$.
No tenemos conocimiento de otros estudios que hayan determinado la asociación entre IMC y talla en niños menores de 3 años. A los 5 años nuestros resultados concuerdan con los de Freedman y cols, que recientemente ${ }^{16}$ analizaron la asociación transversal entre talla y adiposidad en niños entre 3 y 17 años, participantes del "Bogalusa Heart Study”. Ellos mostraron que la proporción de niños con sobrepeso variaba significativamente según la talla, especialmente entre 3 y 10 años de edad, ya que la prevalencia de sobrepeso aumentaba 10 veces desde el quintil 1 al 5 de talla. Estos autores también exploraron esta relación entre los 5 y 18 años desde 1995 al 2000, encontrando que existía una asociación positiva entre IMC y talla en los niños más altos, particularmente entre 5 y 11 años. Otros estudios han mostrado resultados similares $^{17,18}$. Recientemente, Bosy-Westphal y cols ${ }^{19}$, en un estudio transversal de niños y adolescentes alemanes entre 5 y 17 años, demostraron que la asociación positiva entre IMC y estatura era mucho más fuerte en el grupo de niños obesos, con una prevalencia entre 3,2 y 3,7 veces mayor en los niños más altos.

Vale la pena mencionar que en niños pobres la relación positiva entre talla y obesidad puede no 
observarse, tal como lo reportaron Stein y cols ${ }^{20}$ en un estudio de niños guatemaltecos entre 57 y 63 meses.

Es necesario mencionar que al ser la estatura parte del IMC, podría haber una influencia de ésta sobre el indicador ${ }^{21}$. Esto fue confirmado en niños mayores de 5 años, en que las correlaciones entre el IMC y la talla eran aproximadamente de 0,3 hasta los 10 años. Adicionalmente, la encuesta norteamericana NHANES I mostró la influencia que tenían en el IMC las proporciones corporales, mostrando que la correlación de la altura de tronco con IMC era mayor que la de la altura de piernas $^{22}$. Recientemente, Wang ${ }^{23}$, utilizando la encuesta NHANES III, determinó en escolares mayores de 5 años, que existía una correlación entre IMC y talla en hombres hasta los 16 años y en niñas hasta los 11 años.

Stovitz y $\operatorname{cols}^{24}$, en un estudio que tenía como objetivo principal determinar la asociación entre talla e IMC a los 8 años, como predictor del IMC a los 18 años, encontraron que en los cuatro quintiles inferiores de IMC a los 8 años no se observó asociación entre talla e IMC a los 18 años, sin embargo, en el quintil superior de IMC, se observó una asociación positiva y significativa, demostrando que en niños con sobrepeso a los 8 años, aquellos con mayor estatura, tienen mayor probabilidad de tener sobrepeso en la edad adulta.

La mayor fortaleza de este estudio es que fue posible estimar la relación entre talla y obesidad en una cohorte de niños con peso de nacimiento normal que cuenta con datos antropométricos desde 0-5 años. Una limitación importante es que las mediciones antropométricas 0-3 años provienen de los registros de la atención primaria, y no podemos estar seguros si sus estándares de calidad fueron similares a los seguidos por nuestro equipo en las mediciones de los 4 y 5 años. Nosotros abordamos este aspecto examinando la variabilidad de las mediciones y el porcentaje de outliers en cada edad, encontrando que eran similares. Otra limitante se refiere al hecho de que el número de niños evaluados en cada momento difirió en forma importante, por lo que no es del todo descartable un potencial sesgo de selección en nuestros resultados. Sin embargo, nuestros análisis antropométricos demuestran que los sujetos incluidos no difirieron significativamente con aquéllos no incluidos. Adicionalmente, verificamos la consistencia de nuestros resultados (en magnitud y dirección) en una submuestra de 223 sujetos que tenían mediciones en todos los momentos, lo que sugiere que nuestros resultados contarían con una adecuada validez interna.

El hallazgo de que la prevalencia de obesidad fue menor en el grupo con Z TE entre - 1 a 0 , hace reflexionar sobre cuál es el ideal de crecimiento lineal en poblaciones que están experimentando la transición nutricional o post-transición en un ambiente "obesogénico"25. Claramente estos resultados y aquellos de estudios mencionados anteriormente, muestran que el aumento del sobrepeso ha contribuido a disminuir la proporción de niños con talla baja en la etapa preescolar y prepuberal. Si el objetivo es promover un crecimiento saludable y no un exceso de adiposidad, es importante que los esfuerzos para disminuir la talla baja incluyan además el determinar cuál es la contribución relativa de la obesidad al crecimiento lineal.

Agradecimientos: Este estudio fue posible gracias a los Proyectos Fondecyt 1090252 y 709005. Agradecemos sinceramente a los niños y sus madres por participar en estos proyectos. La Dra Corvalán tiene una beca de capacitación de The Wellcome Trust.

\section{Referencias}

1. Lobstein T, Baur L, Uauy R. Obesity in children and young people: a crisis in public health. Obes Rev 2004; 5 (Suppl 1).

2. Junta Nacional de Auxilio Escolar y Becas (JUNAEB) Mapa Nutricional. Consultado en septiembre 2009 www. junaeb.cl.

3. Freedman D, Thornton J, Mei Z, Wang J, Dietz W, Pierson $\mathrm{R}$, et al. Height and adiposity among children. Obes Res 2004; 12: 846-53.

4. Tate A, Dezateux C, Cole T; Millennium Cohort Study Child Health Group. Is infant growth changing? IJO 2006; 30: 1094-6.

5. Murasko J. Socioeconomic status, height, and obesity in children. Econ Hum Biol. 2009; 7:376-386.

6. Buchan I, Bundred P, Kitchiner D, Cole T. Body mass index has risen more steeply in tall than in short 3-year olds: serial cross-sectional surveys 1988-2003. IJO 2007; 31: 23-9.

7. Stanojevic S, Kain J, Uauy R. The association between changes in height and obesity in Chilean preschool 
children: 1996-2004. Obesity 2007; 15: 1012-22.

8. Kain J, Uauy R, Lera L, Taibo M, Albala C. Trends in height and BMI of 6-year-old children during the nutrition transition in Chile. Obes Res 2005; 13: 2178-86.

9. Kain J, Corvalán C, Lera L, Galvan M, Uauy R. Accelerated growth in early life and obesity in preschool Chilean children. Obesity 2009; 17: 1603-8.

10. http://www.junji.cl Junta Nacional de Jardines Infantiles (JUNJI) . Consultado en marzo, 2009.

11. OMS. The WHO Child Growth Standards 2006: Geneva Consultado en enero 2008 http://www.who.int/childgrowth/standards/en/

12. OMS . The WHO 2007 SAS Macro Package. 2007. World Health Organization (WHO): Geneva, Switzerland.

13. Cohen J. Statistical Power Analysis for the Behavioral Sciences. Hillsdale, NJ: Lawrence Erlbaum Assoc 1988.

14. StataCorp LP. Stata Statistical Software: Release 10. 2007. College Station, TX: (http://www2.sas.com/proceedings/ sugi31/204-31.pdf

15. He Q, Karlberg J. BMI in childhood and its association with height gain, timing of puberty and final height. Pediatr Res 2001; 49: 244-51.

16. Freedman D, Khan L, Serdula M, Dietz W, Srinivasan S, Berenson G. Inter-relationships among childhood BMI, childhood height, and adult obesity: the Bogalusa Heart Study. IJO 2004; 28: 10-6.

17. Freedman D, Khan L, Dietz W, Srinivasan S, Berenson G. Relation of childhood height to obesity among adults:
The Bogalusa Heart Study. Pediatrics 2002; 109: E23.

18. Leonard M, Shults J. Wilson B, Tershakovec A, Zernel B. Obesity during childhood and adolescence augments bone mass and bone dimensions. AJCN 2004; 80: $514-$ 23.

19. Bosy-Westphal A, Plachta-Danielzik1 S, Dorhofer P, Muller M. Short stature and obesity: positive association in adults but inverse association in children and adolescents. BJN 2009; 102: 453-61.

20. Stein A, Wang M, Digirolamo A, Hoddinott J, Martorell R, Ramírez-Zea M, et al. Height for age increased while body mass index for age remained stable between 1968 and 2007 among Guatemalan children. J Nutr 2009; 139: 365-9.

21. Poskitt E. Defining childhood obesity: the relative body mass index (BMI) Acta Pediatr 1995; 84: 961-3.

22. Garn S, Leonard W, Hawthorne V. Three limitations of the body mass index. AJCN 1986; 44: 996-7.

23. Wang Y. Epidemiology of childhhod obesity-methodological aspects and guidelines: what is new ?. IJO 2004; 28: S21-8.

24. Stovitz S, Pereira M, Vázquez G, Lytle L, Himes J. The Interaction of Childhood Height and Childhood BMI in the Prediction of Young Adult BMI. Obesity 2008; 16: 2336-234

25. Albala C, Vio F, Kain J, Uauy R. Nutrition transition in Chile. Determinants and consequences. Public Health Nutrition 2002; 5: 123-8. 